\title{
DETERMINATION OF A TYPE OF PERMUTATION BINOMIALS OVER FINITE FIELDS
}

\author{
XIANG-DONG HOU* AND STEPHEN D. LAPPANO
}

\begin{abstract}
Let $f=a \mathrm{x}+\mathrm{x}^{3 q-2} \in \mathbb{F}_{q^{2}}[\mathrm{x}]$, where $a \in \mathbb{F}_{q^{2}}^{*}$. We prove that $f$ is a permutation polynomial of $\mathbb{F}_{q^{2}}$ if and only if one of the following occurs: (i) $q=2^{e}$, e odd, and $a^{\frac{q+1}{3}}$ is a primitive 3 rd root of unity. (ii) $(q, a)$ belongs to a finite set which is determined in the paper.
\end{abstract}

\section{INTRODUCTION}

A polynomial $f \in \mathbb{F}_{q}[\mathrm{x}]$ is called a permutation polynomial $(\mathrm{PP})$ of $\mathbb{F}_{q}$ if it induces a permutation of $\mathbb{F}_{q}$. While permutation monomials of $\mathbb{F}_{q}$ are obvious $\left(a \mathrm{x}^{n}, a \in \mathbb{F}_{q}^{*}\right.$, $\operatorname{gcd}(n, q-1)=1)$, the situation for permutation binomials is much more interesting and challenging. The reason for a binomial to be a PP can be quite nontrivial despite the simple appearance of the binomial. In [3, Carlitz and Wells proved that for fixed integers $e>1$ and $c>0$, when $q$ is large enough and satisfies the conditions $e \mid q-1$ and $\operatorname{gcd}(c, q-1)=1$, there exists $a \in \mathbb{F}_{q}^{*}$ such that $\mathrm{x}^{c}\left(\mathrm{x}^{\frac{q-1}{e}}+a\right)^{k}$ is a $\mathrm{PP}$ of $\mathbb{F}_{q}$ for all $k \geq 0$. (Note that when $k=1$, the $\mathrm{PP}$ is a binomial.) The special cases of this result with $c=k=1$ and $e=2,3$ appeared in 22. Carlitz and Wells' proof of the existence result relies on a bound on the Weil sum of a multiplicative character of $\mathbb{F}_{q}$ [16, [7, Theorem 5.39]. Using the Hasse-Weil bound on the number of degree one places of a function field over $\mathbb{F}_{q}[10$, Theorem V.2.3], Masuda and Zieve [8] were able to make Carlitz-Wells' existence result (with $k=1$ ) more precise. They proved that if $q \geq 4$ and $\frac{q-1}{e}>2 q(\log \log q) / \log q$, then there exists $a \in \mathbb{F}_{q}^{*}$ such that $\mathrm{x}^{c}\left(\mathrm{x}^{\frac{q-1}{e}}+a\right)$ is a PP of $\mathbb{F}_{q}$. Moreover, they obtained an estimate for the number of $a$ 's with this property.

There are also nonexistence results on permutation binomials. Niederreiter and Robinson 9] proved that if there is a PP of $\mathbb{F}_{q}$ of the form $\mathrm{x}^{m}+a \mathrm{x}$, where $m>2$ and $a \in \mathbb{F}_{q}^{*}$, then either $m$ is a power of $p\left(p=\operatorname{char} \mathbb{F}_{q}\right)$ or $q<\left(m^{2}-4 m+6\right)^{2}$. An improvement of this result was obtained by Turnwald [12]: If there is a PP of $\mathbb{F}_{q}$ of the form $\mathrm{x}^{m}+a \mathrm{x}^{n}$, where $m>n>0$ and $a \in \mathbb{F}_{q}^{*}$, then either $\frac{m}{n}$ is a power of $p$ or $q \leq(m-2)^{4}+4 m-4$. For permutation binomials over prime fields, the nonexistence results are stronger. Wan [13] proved that if there is a PP of $\mathbb{F}_{p}$ of the form $\mathrm{x}^{m}+a \mathrm{x}$, where $m>1$ and $a \in \mathbb{F}_{p}^{*}$, then $p-1 \leq(m-1) \operatorname{gcd}(m-1, p-1)$. Turnwald [12] considered $f=\mathrm{x}^{m}+a \mathrm{x}^{n} \in \mathbb{F}_{p}[\mathrm{x}]$, where $m>n>0$ and $a \in \mathbb{F}_{p}^{*}$, and proved that $f$ is a PP of $\mathbb{F}_{p}$ implies $p<m \cdot \max (n, m-n)$. Masuda and Zieve [8] improved Turnwald's bound to $p-1<(m-1) \cdot \max \{n, \operatorname{gcd}(m-n, p-1)\}$.

2000 Mathematics Subject Classification. 11T06, 11 T55.

Key words and phrases. binomial, finite field, Hermite criterion, permutation polynomial.

* Research partially supported by NSA Grant H98230-12-1-0245. 
Let $r \geq 2$. In [2], Carlitz proved that the binomial $\mathrm{x}^{1+\frac{q-1}{2}}+a \mathrm{x}(q$ odd, $a \neq 0)$ cannot be a PP of $\mathbb{F}_{q^{r}}$, and he raised the same question for $\mathrm{x}^{1+\frac{q-1}{3}}+a \mathrm{x}(q \equiv$ $1(\bmod 3), a \neq 0)$. Wan 13 , 14 answered Carlitz's question by showing that $\mathrm{x}^{1+\frac{q-1}{3}}+a \mathrm{x}(q \equiv 1(\bmod 3), a \neq 0)$ cannot be a PP of $\mathbb{F}_{p^{r}}$. Kim and Lee 6 proved that $\mathrm{x}^{1+\frac{q-1}{5}}+a \mathrm{x}(q \equiv 1(\bmod 5), a \neq 0)$ cannot be a $\mathrm{PP}$ of $\mathbb{F}_{q^{r}}$ for $p \neq 2$. More generally, one may consider $\mathrm{x}^{1+\frac{q-1}{m}}+a \mathrm{x} \in \mathbb{F}_{q}[\mathrm{x}]$, where $q \equiv 1(\bmod m), m \geq 2$, $a \neq 0$. Clearly, if $m=\frac{q-1}{p^{i}-1}$, where $\mathbb{F}_{p^{i}} \subset \mathbb{F}_{q}$, then $\mathrm{x}^{1+\frac{q-1}{m}}+a \mathrm{x}=\mathrm{x}^{p^{i}}+a \mathrm{x}$, which is a $\mathrm{PP}$ of $\mathbb{F}_{q^{r}}$ if and only if $(-a)^{\left(q^{r}-1\right) /\left(p^{i}-1\right)} \neq 1$. When $1+\frac{q-1}{m}$ is not a power of $p$, it is not known if the binomial can be a PP of $\mathbb{F}_{q^{r}}$.

Let $f=\mathrm{x}^{m}+a \mathrm{x}^{n} \in \mathbb{F}_{q}[\mathrm{x}]$, where $m>n>0$ and $a \in \mathbb{F}_{q}^{*}$. The conditions that make $f$ a PP of $\mathbb{F}_{q}$ are encoded in a simple set of parameters $m, n, q, a$ in a mysterious way that is not well understood on the whole. However, when $m$ and $n$ take certain particular forms, necessary and sufficient conditions for $f$ to be a PP of $\mathbb{F}_{q}$ have been found. Niederreiter and Robinson $\left[9\right.$ proved that $\mathrm{x}^{\frac{q+1}{2}}+a \mathrm{x} \in \mathbb{F}_{q}[x]$ ( $q$ odd, $a \in \mathbb{F}_{q}^{*}$ ) is a PP of $\mathbb{F}_{q}$ if and only if $a^{2}-1$ is a square in $\mathbb{F}_{q}^{*}$; also see [2]. Akbary and Wang [1] considered binomials of the form $f=\mathrm{x}^{r}\left(1+\mathrm{x}^{e s}\right)$, where $e, r, s$ are positive integers such that $s \mid q-1, \operatorname{gcd}(r, s)=1, \operatorname{gcd}\left(2 e, \frac{q-1}{s}\right)=1$. They found sufficient conditions for $f$ to be a PP of $\mathbb{F}_{q}$ in terms of the period of the generalized Lucas sequence. The conditions are not entirely explicit, but their special cases do give explicit classes of permutation binomials of $\mathbb{F}_{q}$. The sufficient conditions in [1] were later extended by Wang [15] to conditions that are both necessary and sufficient. Zieve [17] considered $f=\mathrm{x}^{m}+a \mathrm{x}^{n} \in \mathbb{F}_{q}[\mathrm{x}]$, where $m>n>0$ and $a \in \mathbb{F}_{q}^{*}$, under the assumption that $\left\{\eta+\frac{1}{\eta}: \eta \in \mu_{2 d}\right\} \subset \mu_{s}$, where $s=\operatorname{gcd}(m-n, q-1)$, $d=\frac{q-1}{s}$ and $\mu_{s}=\left\{x \in \mathbb{F}_{q}: x^{s}=1\right\}$. In this setting, it was shown that $f$ is a PP of $\mathbb{F}_{q}$ if and only $-a \notin \mu_{d}, \operatorname{gcd}(n, s)=1$ and $\operatorname{gcd}(2 d, m+n) \leq 2$. (This characterization implies the aforementioned sufficient conditions in [1]; see [17].)

Recently, we determined all PPs of $\mathbb{F}_{q^{2}}$ of the form $a \mathrm{x}+\mathrm{x}^{2 q-1}\left(a \in \mathbb{F}_{q^{2}}^{*}\right)$ [4] [5]. The techniques introduced there are applicable to binomials of similar types. In the present paper, we determine all PPs of $\mathbb{F}_{q^{2}}$ of the form $a \mathrm{x}+\mathrm{x}^{3 q-2}$. Our main result is the following.

Theorem 1.1. Let $f=a \mathrm{x}+\mathrm{x}^{3 q-2} \in \mathbb{F}_{q^{2}}[\mathrm{x}]$, where $a \in \mathbb{F}_{q^{2}}^{*}$. Then $f$ is a PP of $\mathbb{F}_{q^{2}}$ if and only if one of the following occurs.

(i) $q=2^{2 k+1}$ and $a^{\frac{q+1}{3}}$ is a primitive $3 r d$ root of unity.

(ii) $q=5$ and $a^{2}$ is a root of $(\mathrm{x}+1)(\mathrm{x}+2)(\mathrm{x}-2)\left(\mathrm{x}^{2}-\mathrm{x}+1\right)$.

(iii) $q=2^{3}$ and $a^{3}$ is a root of $\mathrm{x}^{3}+\mathrm{x}+1$.

(iv) $q=11$ and $a^{4}$ is a root of $(\mathrm{x}-5)(\mathrm{x}+2)\left(\mathrm{x}^{2}-\mathrm{x}+1\right)$.

(v) $q=17$ and $a^{6}=4,5$.

(vi) $q=23$ and $a^{8}=-1$.

(vii) $q=29$ and $a^{10}=-3$.

Note. Shortly after the submission of the first vision of this paper, we were informed by M. Zieve of two very recent papers [11] by Tu, Zeng, Hu and Li and [18] by Zieve in the arXiv. We remark that under the assumption that $a$ is a $(q+1)$ st root of unity, Theorem 1.1 (i), which is the only relevant case under this assumption, follows from [18, Corollary 5.3]. If, in addition, $q$ is assumed to be even, then [11, Theorem 1] also gives sufficiency part of Theorem 1.1 (i). 


\section{Preliminaries}

Let $f=a \mathrm{x}+\mathrm{x}^{3 q-2} \in \mathbb{F}_{q^{2}}[\mathrm{x}]$, where $a \in \mathbb{F}_{q^{2}}^{*}$, and let $0 \leq \alpha, \beta \leq q-1$. We have

$$
\begin{aligned}
\sum_{x \in \mathbb{F}_{q^{2}}} f(x)^{\alpha+\beta q} & =\sum_{x \in \mathbb{F}_{q^{2}}^{*}}\left(a x+x^{3 q-2}\right)^{\alpha}\left(a^{q} x^{q}+x^{3-2 q}\right)^{\beta} \\
& =\sum_{x \in \mathbb{F}_{q^{2}}^{*}} \sum_{i, j}\left(\begin{array}{c}
\alpha \\
i
\end{array}\right)(a x)^{\alpha-i} x^{(3 q-2) i}\left(\begin{array}{c}
\beta \\
j
\end{array}\right)\left(a^{q} x^{q}\right)^{\beta-j} x^{(3-2 q) j} \\
& =a^{\alpha+\beta q} \sum_{i, j}\left(\begin{array}{c}
\alpha \\
i
\end{array}\right)\left(\begin{array}{c}
\beta \\
j
\end{array}\right) a^{-i-j q} \sum_{x \in \mathbb{F}_{q^{2}}^{*}} x^{\alpha+\beta q+3(q-1)(i-j)} .
\end{aligned}
$$

The inner sum is 0 unless $\alpha+\beta q \equiv 1(\bmod q-1)$, i.e., $\alpha+\beta=q-1$.

Assume $0 \leq \alpha \leq q-1$ and $\beta=q-1-\alpha$ in (2.1). We have

$$
\begin{aligned}
& \sum_{x \in \mathbb{F}_{q^{2}}} f(x)^{\alpha+(q-1-\alpha) q} \\
= & a^{(\alpha+1)(1-q)} \sum_{i, j}\left(\begin{array}{c}
\alpha \\
i
\end{array}\right)\left(\begin{array}{c}
q-1-\alpha \\
j
\end{array}\right) a^{-i-j q} \sum_{x \in \mathbb{F}_{q^{*}}^{*}} x^{(q-1)[-\alpha-1+3(i-j)]} \\
= & -a^{(\alpha+1)(1-q)} \sum_{-\alpha-1+3(i-j) \equiv 0(\bmod q+1)}\left(\begin{array}{c}
\alpha \\
i
\end{array}\right)\left(\begin{array}{c}
q-1-\alpha \\
j
\end{array}\right) a^{-i-j q} .
\end{aligned}
$$

As $i$ runs over the interval $[0, \alpha]$ and $j$ over the interval $[0, q-1-\alpha]$, the range of $-\alpha-1+3(i-j)$ is

$$
I_{\alpha}:=[2 \alpha+2-3 q, \alpha-1]
$$

Thus we have

$$
\sum_{x \in \mathbb{F}_{q^{2}}} f(x)^{\alpha+(q-1-\alpha) q}=-a^{(\alpha+1)(1-q)} S_{q}(\alpha, a)
$$

where

$$
S_{q}(\alpha, a)=\sum_{-\alpha-1+3(i-j) \in I_{\alpha} \cap(q+1) \mathbb{Z}}\left(\begin{array}{c}
\alpha \\
i
\end{array}\right)\left(\begin{array}{c}
q-1-\alpha \\
j
\end{array}\right) a^{-i-j q} .
$$

By Hermite's criterion, $f$ is a $\mathrm{PP}$ of $\mathbb{F}_{q^{2}}$ if and only if 0 is the only root of $f$ and

$$
S_{q}(\alpha, a)=0 \text { for all } 0 \leq \alpha \leq q-1 .
$$

Lemma 2.1. If $f$ is a PP of $\mathbb{F}_{q^{2}}$, then $q+1 \equiv 0(\bmod 3)$.

Proof. Assume $f$ is a $\mathrm{PP}$ of $\mathbb{F}_{q^{2}}$ and assume $q \geq 3$. If $q=3$, the only multiple of $q+1$ in $I_{0}=[2-3 q,-1]$ is $-(q+1)$. By (2.6) (with $\alpha=0$ ), we have

$$
0=\sum_{-1-3 j=-(q+1)}\left(\begin{array}{c}
q-1 \\
j
\end{array}\right) a^{-j q}=-a^{-q},
$$

which is a contradiction. Now assume $q \geq 4$. The multiples of $q+1$ in $I_{0}=$ $[2-3 q,-1]$ are $-2(q+1)$ and $-(q+1)$. By (2.6) (with $\alpha=0)$,

(2.7) $0=\sum_{-1-3 j=-2(q+1),-(q+1)}\left(\begin{array}{c}q-1 \\ j\end{array}\right) a^{-j q}=\left(\begin{array}{c}q-1 \\ \frac{2 q+1}{3}\end{array}\right)^{*} a^{-\frac{2 q+1}{3} q}+\left(\begin{array}{c}q-1 \\ \frac{q}{3}\end{array}\right)^{*} a^{-\frac{q}{3}}$, 
where

$$
\left(\begin{array}{l}
m \\
n
\end{array}\right)^{*}= \begin{cases}\left(\begin{array}{l}
m \\
n
\end{array}\right) & \text { if } n \in \mathbb{N} \\
0 & \text { otherwise. }\end{cases}
$$

If, to the contrary, $q+1 \not \equiv 0(\bmod 3)$, then exactly one of $\left(\begin{array}{c}q-1 \\ \frac{2 q+1}{3}\end{array}\right)^{*}$ and $\left(\begin{array}{c}q-1 \\ \frac{q}{3}\end{array}\right)^{*}$ is nonzero, and hence (2.7) cannot hold.

Remark 2.2. Assume $q+1 \equiv 0(\bmod 3)$.

(i) 0 is the only root of $f$ in $\mathbb{F}_{q^{2}}$ if and only if $a^{\frac{q+1}{3}} \neq 1$. (Note that $(-1)^{\frac{q+1}{3}}=$ 1.)

(ii) For $b \in \mathbb{F}_{q^{2}}^{*}$, we have $f(b \mathrm{x})=b^{3 q-2}\left(b^{3(1-q)} a \mathrm{x}+\mathrm{x}^{3 q-2}\right)$. Thus if $f$ is a PP of $\mathbb{F}_{q^{2}}$ for $a=a_{0}$, the same is true for $a=\epsilon a_{0}$, where $\epsilon \in \mathbb{F}_{q^{2}}, \epsilon^{\frac{q+1}{3}}=1$.

Lemma 2.3. Assume $q+1 \equiv 0(\bmod 3), \alpha>0, \alpha+1 \equiv 0(\bmod 3), q \geq 2 \alpha+4$. Then

$S_{q}(\alpha, a)=(-a)^{\frac{q+1}{3}} q \sum_{i=0}^{\alpha}(-1)^{i}\left(\begin{array}{c}\alpha \\ i\end{array}\right)\left[\left(\begin{array}{c}i+\frac{2 \alpha-1}{3} \\ \alpha\end{array}\right) v^{3 i}+\left(\begin{array}{c}i+\frac{2 \alpha}{3} \\ \alpha\end{array}\right) v^{3 i+1}+\left(\begin{array}{c}i+\frac{2 \alpha+1}{3} \\ \alpha\end{array}\right) v^{3 i+2}\right]$,

where $S_{q}(\alpha, a)$ is defined in (2.5) and $v=a^{-\frac{q+1}{3}}$.

Proof. Since $\alpha>0$ and $q \geq 2 \alpha+4$, the multiples of $q+1$ in $I_{\alpha}=[2 \alpha+2-3 q, 2 \alpha-1]$ are $-2(q+1),-(q+1), 0$. We have

LHS of (2.8)

$$
\begin{aligned}
& =\sum_{-\alpha-1+3(i-j)=-2(q+1),-(q+1), 0}\left(\begin{array}{c}
\alpha \\
i
\end{array}\right)\left(\begin{array}{c}
q-1-\alpha \\
j
\end{array}\right) a^{-i-j q} \\
& =\sum_{i=0}^{\alpha}\left(\begin{array}{l}
\alpha \\
i
\end{array}\right) \sum_{l=0}^{2}\left(\begin{array}{c}
-1-\alpha \\
\frac{1}{3}(l(q+1)-\alpha-1)+i
\end{array}\right) a^{-i-\left[\frac{1}{3}(l(q-1)-\alpha-1)+i\right] q} \\
& =\sum_{i=0}^{\alpha}\left(\begin{array}{l}
\alpha \\
i
\end{array}\right) \sum_{l=0}^{2}(-1)^{\frac{1}{3}(l(q+1)-\alpha-1)+i}\left(\begin{array}{c}
\frac{1}{3}(l(q+1)-\alpha-1)+i+\alpha \\
\alpha
\end{array}\right) a^{\frac{\alpha+1}{3} q-\frac{q+1}{3}(l+3 i)} \\
& \left(\left(\begin{array}{c}
-m \\
n
\end{array}\right)=(-1)^{n}\left(\begin{array}{c}
n+m-1 \\
m-1
\end{array}\right) \text { for } m, n \in \mathbb{N}\right) \\
& =(-a)^{\frac{q+1}{3}} q \sum_{i=0}^{\alpha}(-1)^{i}\left(\begin{array}{c}
\alpha \\
i
\end{array}\right) \sum_{l=0}^{2}\left(\begin{array}{c}
i+\frac{2 \alpha-1+l}{3} \\
\alpha
\end{array}\right) v^{3 i+l} \text {. }
\end{aligned}
$$

\section{Proof of Theorem 1.1}

Lemma 3.1. Assume $q+1 \equiv 0(\bmod 3)$ and $y:=a^{\frac{q+1}{3}}$ is a primitive $3 r d$ root of unity. Then for $1 \leq s \leq q^{2}-2$,

$$
\sum_{x \in \mathbb{F}_{q^{2}}} f(x)^{s}= \begin{cases}a^{-\frac{1}{6}(q+1)(3 q-2)}(1+y) & \text { if } q \text { is odd and } s=\frac{q^{2}-1}{2} \\ 0 & \text { otherwise. }\end{cases}
$$


Proof. By the observation after (2.1), we only have to consider $s=\alpha+(q-1-\alpha) q$, $0 \leq \alpha \leq q-1$. By (2.4), we may further assume $\alpha+1 \equiv 0(\bmod 3)$. There is nothing to prove if $q=2$. Thus we assume $q>3$.

$1^{\circ}$ We claim that $I_{\alpha}=[2 \alpha+2-3 q, 2 \alpha-1]$ contains exactly three consecutive multiples of $q+1$ unless $\alpha=\frac{q-1}{2}$ ( $q$ odd); in the latter case, $I_{\alpha}=[-2 q+1, q-2]$ contains exactly two multiples of $q+1$ which are $-(q+1)$ and 0 .

The length of $I_{\alpha}$ is $2 \alpha-1-(2 \alpha+2-3 q)=3(q-1)$. Since $2 q<3(q-1)<3(q+1)$, there are at least 2 and at most 3 multiples of $q+1$ in $I_{\alpha}$. Let $k(q+1),(k+1)(q+1) \in$ $I_{\alpha}$. If $I_{\alpha}=[2 \alpha+2-3 q, 2 \alpha-1]$ contains only two multiples of $q+1$, then

$$
\left\{\begin{array}{l}
2 \alpha+2-3 q \geq k(q+1)-q, \\
2 \alpha-1 \leq(k+1)(q+1)+q .
\end{array}\right.
$$

(See Figure 1, )

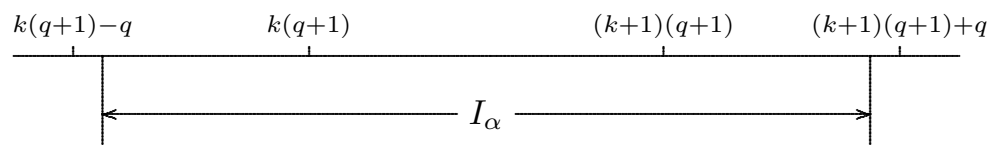

Figure 1. Proof of (3.2)

Since $2 \alpha+2-3 q, 2 \alpha-1 \equiv 0(\bmod 3)$, we have

$$
\left\{\begin{array}{l}
2 \alpha+2-3 q \geq k(q+1)-q+2 \\
2 \alpha-1 \leq(k+1)(q+1)+q-2
\end{array}\right.
$$

Taking the difference of the two inequalities in (3.3), we conclude that

$$
\left\{\begin{array}{l}
2 \alpha+2-3 q=k(q+1)-q+2 \\
2 \alpha-1=(k+1)(q+1)+q-2
\end{array}\right.
$$

Since $0 \leq \alpha \leq q-1$, we must have $k=-1$. Thus $\alpha=\frac{q-1}{2}$. 
$2^{\circ}$ First assume $\alpha \neq \frac{q-1}{2}$. Let $I_{\alpha} \cap(q+1) \mathbb{Z}=(q+1) L$, where $L$ is a set of three consecutive integers. Then we have

$$
\begin{aligned}
& S_{q}(\alpha, a)=\sum_{-\alpha-1+3(i-j) \in I_{\alpha} \cap(q+1) \mathbb{Z}}\left(\begin{array}{c}
\alpha \\
i
\end{array}\right)\left(\begin{array}{c}
q-1-\alpha \\
j
\end{array}\right) a^{-i-j q} \\
& =\sum_{l \in L} \sum_{-\alpha-1+3(i-j)=l(q+1)}\left(\begin{array}{c}
\alpha \\
i
\end{array}\right)\left(\begin{array}{c}
q-1-\alpha \\
j
\end{array}\right) a^{-i-j q} \\
& =\sum_{l \in L} \sum_{i-j=\frac{1}{3}[\alpha+1+l(q+1)]}\left(\begin{array}{c}
\alpha \\
i
\end{array}\right)\left(\begin{array}{c}
q-1-\alpha \\
j
\end{array}\right) a^{-i+j} \quad\left(\text { since } a^{q+1}=1\right) \\
& =\sum_{l \in L} a^{-\frac{1}{3}[\alpha+1+l(q+1)]} \sum_{i-j=\frac{1}{3}[\alpha+1+l(q+1)]}\left(\begin{array}{c}
\alpha \\
\alpha-i
\end{array}\right)\left(\begin{array}{c}
q-1-\alpha \\
j
\end{array}\right) \\
& =a^{-\frac{1}{3}(\alpha+1)} \sum_{l \in L} y^{-l} \sum_{\alpha-i+j=\frac{1}{3}[2 \alpha-1-l(q+1)]}\left(\begin{array}{c}
\alpha \\
\alpha-i
\end{array}\right)\left(\begin{array}{c}
q-1-\alpha \\
j
\end{array}\right) \\
& =a^{-\frac{1}{3}(\alpha+1)} \sum_{l \in L} y^{-l}\left(\begin{array}{c}
q-1 \\
\frac{1}{3}[2 \alpha-1-l(q+1)]
\end{array}\right) \\
& =-a^{-\frac{1}{3}(\alpha+1)} \sum_{l \in L} y^{-l} \\
& =0 \text {. }
\end{aligned}
$$

In the next-to-last step, we have $\left(\begin{array}{c}q-1 \\ \frac{1}{3}[2 \alpha-1-l(q+1)]\end{array}\right)=(-1)^{\frac{1}{3}[2 \alpha-1-l(q+1)]}=-1$ since $0 \leq \frac{1}{3}[2 \alpha-1-l(q+1)] \leq \frac{1}{3}[2 \alpha-1-(2 \alpha+2-3 q)]=q-1$. Combining (2.4) and (3.4) gives $\sum_{x \in \mathbb{F}_{q^{2}}} f(x)^{s}=0$.

$3^{\circ}$ Now assume $\alpha=\frac{q-1}{2}$. By the calculation in (3.4), we have

$$
S_{q}(\alpha, a)=-a^{-\frac{1}{3}(\alpha+1)} \sum_{l=-1,0} y^{-l}=-a^{-\frac{1}{6}(q+1)}(1+y) .
$$

Combining (2.4) and (3.5) gives

$$
\sum_{x \in \mathbb{F}_{q^{2}}} f(x)^{s}=a^{\frac{q+1}{2}(1-q)-\frac{q+1}{6}}(1+y)=a^{-\frac{1}{6}(q+1)(3 q-2)}(1+y) .
$$

Proof of Theorem 1.1. $(\Leftarrow)$ (ii) - (vii) are sporadic cases with small $q$. Using a computer, it is easy to verify that $f$ is a PP in each of these cases. Now assume (i). By Lemma 3.1, we have $\sum_{x \in \mathbb{F}_{q^{2}}} f(x)^{s}=0$ for all $1 \leq s \leq q^{2}-2$. Also note from Remark 2.2 (i) that 0 is the only root of $f$ in $\mathbb{F}_{q^{2}}$. Thus $f$ is a PP of $\mathbb{F}_{q^{2}}$.

$(\Rightarrow)$ Assume that $f$ is a $\mathrm{PP}$ of $\mathbb{F}_{q^{2}}$. By Lemma 2.1, we have $q+1 \equiv 0(\bmod 3)$. Let $y=a^{\frac{q+1}{3}}$. If $y$ is a primitive 3rd root of unity, by Lemma 3.1, $q$ must be even, i.e., $q=2^{2 k+1}$, and we have case (i). Now assume that $y^{2}+y+1 \neq 0$. We show that one of the cases (ii) - (vii) occurs.

The sum in the right side of (2.8) is a polynomial in $y^{-1}(=v)$ which can be easily computed for small $\alpha$ with computer assistance. For $\alpha=2,5,8,11,14$, we 
DETERMINATION OF A TYPE OF PERMUTATION BINOMIALS OVER FINITE FIELDS 7

find that

$$
S_{q}(\alpha, a)=(-a)^{\frac{\alpha+1}{3}} y^{-3 \alpha-2}\left(y^{2}+y+1\right) \begin{cases}3^{-2} g_{2}(y) & \text { if } \alpha=2, q \geq 8, \\ 3^{-6} g_{5}(y) & \text { if } \alpha=5, q \geq 14 \\ 3^{-10} g_{8}(y) & \text { if } \alpha=8, q \geq 20 \\ 3^{-15} g_{11}(y) & \text { if } \alpha=11, q \geq 26 \\ 3^{-19} g_{14}(y) & \text { if } \alpha=14, q \geq 32\end{cases}
$$

where

$$
\begin{aligned}
& g_{2}(\mathrm{x})=2 \mathrm{x}^{5}+3 \mathrm{x}^{4}-23 \mathrm{x}^{3}-8 \mathrm{x}^{2}-9 \mathrm{x}+44, \\
& g_{5}(\mathrm{x})=-14 \mathrm{x}^{14}-8 \mathrm{x}^{13}+22 \mathrm{x}^{12}-469 \mathrm{x}^{11}-1093 \mathrm{x}^{10}+8852 \mathrm{x}^{9}+6801 \mathrm{x}^{8}+10527 \mathrm{x}^{7} \\
& -61068 \mathrm{x}^{6}-18619 \mathrm{x}^{5}-25033 \mathrm{x}^{4}+120197 \mathrm{x}^{3}+13516 \mathrm{x}^{2}+16822 \mathrm{x}-71162, \\
& g_{8}(\mathrm{x})=130 \mathrm{x}^{23}+57 \mathrm{x}^{22}-187 \mathrm{x}^{21}+4082 \mathrm{x}^{20}+3585 \mathrm{x}^{19}-7667 \mathrm{x}^{18}+156234 \mathrm{x}^{17} \\
& +453573 \mathrm{x}^{16}-3916551 \mathrm{x}^{15}-4144622 \mathrm{x}^{14}-7594467 \mathrm{x}^{13}+48939959 \mathrm{x}^{12} \\
& +25221008 \mathrm{x}^{11}+39342423 \mathrm{x}^{10}-213366911 \mathrm{x}^{9}-61811112 \mathrm{x}^{8}-88032825 \mathrm{x}^{7} \\
& +422650317 \mathrm{x}^{6}+66303028 \mathrm{x}^{5}+88882095 \mathrm{x}^{4}-389019163 \mathrm{x}^{3}-25886212 \mathrm{x}^{2} \\
& -33211905 \mathrm{x}+135094180 \\
& g_{11}(\mathrm{x})=-3952 \mathrm{x}^{32}-1522 \mathrm{x}^{31}+5474 \mathrm{x}^{30}-139802 \mathrm{x}^{29}-89324 \mathrm{x}^{28}+229126 \mathrm{x}^{27} \\
& -3943602 \mathrm{x}^{26}-4392909 \mathrm{x}^{25}+8336511 \mathrm{x}^{24}-180820302 \mathrm{x}^{23}-605825169 \mathrm{x}^{22} \\
& +5521784781 \mathrm{x}^{21}+7111655988 \mathrm{x}^{20}+14607372831 \mathrm{x}^{19}-101269369227 \mathrm{x}^{18} \\
& -69095625624 \mathrm{x}^{17}-119477261853 \mathrm{x}^{16}+705650100129 \mathrm{x}^{15}+303870716124 \mathrm{x}^{14} \\
& +475920749355 \mathrm{x}^{13}-2503382174319 \mathrm{x}^{12}-706243777836 \mathrm{x}^{11}-1034492806725 \mathrm{x}^{10} \\
& +4972469163636 \mathrm{x}^{9}+898579001889 \mathrm{x}^{8}+1253008322595 \mathrm{x}^{7}-5598768742164 \mathrm{x}^{6} \\
& -591556509206 \mathrm{x}^{5}-794043854630 \mathrm{x}^{4}+3339003167188 \mathrm{x}^{3}+157572058982 \mathrm{x}^{2} \\
& +205140400010 \mathrm{x}-819352075360 \text {, } \\
& g_{14}(\mathrm{x})=41800 \mathrm{x}^{41}+14895 \mathrm{x}^{40}-56695 \mathrm{x}^{39}+1691000 \mathrm{x}^{38}+905631 \mathrm{x}^{37}-2596631 \mathrm{x}^{36} \\
& +47250150 \mathrm{x}^{35}+37894401 \mathrm{x}^{34}-85144551 \mathrm{x}^{33}+1395800990 \mathrm{x}^{32}+1826164521 \mathrm{x}^{31} \\
& -3221965511 \mathrm{x}^{30}+75566097190 \mathrm{x}^{29}+281332431561 \mathrm{x}^{28}-2683745985685 \mathrm{x}^{27} \\
& -3976231919076 \mathrm{x}^{26}-8901790877799 \mathrm{x}^{25}+65232090577890 \mathrm{x}^{24}+53701334712609 \mathrm{x}^{23} \\
& +100487514543597 \mathrm{x}^{22}-632854611825486 \mathrm{x}^{21}-347885978019711 \mathrm{x}^{20} \\
& -586551837541203 \mathrm{x}^{19}+3307822221633594 \mathrm{x}^{18}+1283881108529889 \mathrm{x}^{17} \\
& +2015859062567817 x^{16}-10419893389315746 x^{15}-2892546806289271 x^{14} \\
& -4307726185011783 \mathrm{x}^{13}+20728564105915330 \mathrm{x}^{12}+4054215382726378 \mathrm{x}^{11} \\
& +5793391583605092 \mathrm{x}^{10}-26245535590106350 \mathrm{x}^{9}-3451745974770042 \mathrm{x}^{8} \\
& -4770402189292728 x^{7}+20520594631893930 x^{6}+1634454816505198 x^{5} \\
& +2197118421394272 \mathrm{x}^{4}-9034762128135730 \mathrm{x}^{3}-330180086243950 \mathrm{x}^{2} \\
& -433563200685120 x+1713531735146800 \text {. }
\end{aligned}
$$


Combining (2.6) and (3.6), we have

$$
\begin{cases}g_{2}(y)=0 & \text { if } q \geq 8 \\ g_{5}(y)=0 & \text { if } q \geq 14 \\ g_{8}(y)=0 & \text { if } q \geq 20 \\ g_{11}(y)=0 & \text { if } q \geq 26 \\ g_{14}(y)=0 & \text { if } q \geq 32\end{cases}
$$

When $q<14$, a quick computer search produces cases (ii) - (iv). So we assume $q \geq 14$. Next we compute the resultant of $g_{2}$ and $g_{5}$ :

$$
R\left(g_{2}, g_{5}\right)=2^{5} \cdot 3^{35} \cdot 17^{2} \cdot 23 \cdot 29 \cdot 103 \cdot 16069 .
$$

By (3.7) and the fact that $q+1 \equiv 0(\bmod 3)$, we must have $p=\operatorname{char} \mathbb{F}_{q} \in$ $\{2,17,23,29\}$.

When $p=2$, we have $q \geq 32$. In this case, $\operatorname{gcd}\left(g_{2}, g_{5}, g_{8}\right)=\mathrm{x}$, which is a contradiction to 3.7).

When $p=17$, we find that $\operatorname{gcd}\left(g_{2}, g_{5}, g_{8}\right)=1$. By (3.7), we must have $q=17$. A computer search results in case $(\mathrm{v})$.

When $p=23, \operatorname{gcd}\left(g_{2}, g_{5}, g_{8}\right)=\mathrm{x}+1$, and $g_{11}(-1)=12 \neq 0$. By (3.7), we must have $q=23$. A computer search results in case (vi).

When $p=29, \operatorname{gcd}\left(g_{2}, g_{5}, g_{8}\right)=\mathrm{x}+10$, and $g_{11}(-10)=0, g_{14}(-10)=2 \neq 0$. By (3.7), we must have $q=29$. A computer search results in case (vii).

\section{REFERENCES}

[1] A. Akbary and Q. Wang, A generalized Lucas sequence and permutation binomials, Proc. Amer. Math. Soc. 134 (2006), $15-22$.

[2] L. Carlitz, Some theorems on permutation polynomials, Bull. Amer. Math. Soc. 68 (1962) $120-122$.

[3] L. Carlitz and C. Wells, The number of solutions of a special system of equations in a finite field, Acta Arith. 12 (1966/1967) 77 - 84.

[4] X. Hou, A class of permutation binomials over finite fields, J. Number Theory 133 (2013), $3549-3558$.

[5] X. Hou, Determination of a type of permutation trinomials over finite fields, II, preprint.

[6] S. Y. Kim and J. B. Lee, Permutation polynomials of the type $x^{1+((q-1) / m)}+a x$, Commun. Korean Math. Soc. 10 (1995), 823 - 829.

[7] R. Lidl and H. Niederreiter, Finite Fields, 2nd ed., Cambridge Univ. Press, Cambridge, 1997.

[8] A. M. Masuda and M. E. Zieve, Permutation binomials over finite fields, Trans. Amer. Math. Soc. 361 (2009), $4169-4180$.

[9] H. Niederreiter and K. H. Robinson, Complete mappings of finite fields, J. Austral. Math. Soc. Ser. A 33 (1982), $197-212$.

[10] H. Stichtenoth, Algebraic Function Fields and Codes, Universitext. Springer-Verlag, Berlin, 1993.

[11] Z. Tu, X. Zeng, L. Hu, C. Li, A class of binomial permutation polynomials, arXiv:1310.0337, 2013.

[12] G. Turnwald, Permutation polynomials of binomial type, Contributions to General Algebra, 6, 281 - 286, Hölder-Pichler-Tempsky, Vienna, 1988.

[13] D. Wan, Permutation polynomials over finite fields, Acta Math. Sinica (N.S.) 3 (1987), 1 5.

[14] D. Wan, Permutation binomials over finite fields, Acta Math. Sinica (N.S.) 10 (1994), Special Issue, $30-35$.

[15] Q. Wang, Cyclotomic mapping permutation polynomials over finite fields, in Sequences, Subsequences, and Consequences, S.W. Golomb, G. Gong, T. Helleseth, H.-Y. Song, (Eds.), pp. 119 - 128, Lecture Notes in Comput. Sci., vol. 4893, Springer, Berlin, 2007. 
[16] A. Weil, On the Riemann hypothesis in function fields, Proc. Nat. Acad. Sci. U. S. A. 27 (1941), $345-347$.

[17] M. E. Zieve, On some permutation polynomials over $\mathbb{F}_{q}$ of the form $x^{r} h\left(x^{(q-1) / d}\right)$, Proc. Amer. Math. Soc. 137 (2009), 2209 - 2216.

[18] M. E. Zieve, Permutation polynomials on $\mathbb{F}_{q}$ induced from Rédei function bijections on subgroups of $\mathbb{F}_{q}^{*}, \operatorname{arXiv:1310.0776} 2013$.

Department of Mathematics and Statistics, University of South Florida, Tampa, FL 33620

E-mail address: xhou@usf.edu

Department of Mathematics and Statistics, University of South Florida, Tampa, FL 33620

E-mail address: slappano@mail.usf.edu 